\title{
Revisiting scalar quark hidden sector in light of 750-GeV diphoton resonance
}

\author{
Cheng-Wei Chiang, ${ }^{a, b, c}$ Masahiro Ibe ${ }^{d, e}$ and Tsutomu T. Yanagida ${ }^{d}$ \\ ${ }^{a}$ Center for Mathematics and Theoretical Physics and Department of Physics, \\ National Central University, \\ Taoyuan, 32001 Taiwan, Republic of China \\ ${ }^{b}$ Institute of Physics, Academia Sinica, \\ Taipei, 11529 Taiwan, Republic of China \\ ${ }^{c}$ Physics Division, National Center for Theoretical Sciences, \\ Hsinchu, 30013 Taiwan, Republic of China \\ ${ }^{d}$ Kavli IPMU (WPI), UTIAS, University of Tokyo, \\ Kashiwa, Chiba 277-8583, Japan \\ e ICRR, University of Tokyo, \\ Kashiwa, Chiba 277-8582, Japan \\ E-mail: chengwei@ncu.edu.tw, ibe@icrr.u-tokyo.ac.jp, \\ tsutomu.tyanagida@ipmu.jp
}

Abstract: We revisit the model of a $C P$-even singlet scalar resonance proposed in arXiv:1507.02483, where the resonance appears as the lightest composite state made of scalar quarks participating in hidden strong dynamics. We show that the model can consistently explain the excess of diphoton events with an invariant mass around $750 \mathrm{GeV}$ reported by both the ATLAS and CMS experiments. We also discuss the nature of the charged composite states in the $\mathrm{TeV}$ range which accompany to the neutral scalar. Due to inseparability of the dynamical scale and the mass of the resonance, the model also predicts signatures associated with the hidden dynamics such as leptons, jets along with multiple photons at future collider experiments. We also associate the $\mathrm{TeV}$-scale dynamics behind the resonance with an explanation of dark matter.

Keywords: Beyond Standard Model, Technicolor and Composite Models, GUT

ARXIV EPRINT: 1512.08895 


\section{Contents}

1 Introduction 1

$2 \quad$ A scalar resonance from hidden dynamics 2

$\begin{array}{llr}3 & \text { Discussions and conclusions } & 8\end{array}$

\section{Introduction}

Recently, both the ATLAS and CMS Collaborations reported intriguing excess events in the search for a high-mass resonance decaying into diphotons in $13-\mathrm{TeV} p p$ collisions $[1,2]$. The excess peaks at the diphoton invariant mass around $750 \mathrm{GeV}$, with significances being $3.6 \sigma$ and $2.6 \sigma$ by using $3.2 \mathrm{fb}^{-1}$ and $2.6 \mathrm{fb}^{-1}$ of data, respectively. Using the model of a narrow scalar resonance, these local significances are reproduced when its production cross section times the branching ratio into diphotons are

$$
\begin{array}{ll}
\sigma(p p \rightarrow S) \times B r(S \rightarrow \gamma \gamma)=(10 \pm 3) \mathrm{fb} & (\text { ATLAS }) \\
\sigma(p p \rightarrow S) \times \operatorname{Br}(S \rightarrow \gamma \gamma)=(6 \pm 3) \mathrm{fb} & (\mathrm{CMS}),
\end{array}
$$

respectively [3]. (See also refs. [4-15] for phenomenological analyses of the resonance.)

After the reports, a plethora of models have been discussed to account for the signals. Among them, models of (pseudo) scalar resonances originating from hidden strong dynamics have gathered particular attention, with its production at the LHC and decay into photons being explained via the gauge interactions of the constituents of the singlet composite state [16-24]. In this paper, we want to point out that an existing model proposed in ref. [25] can consistently account for the diphoton signal while evading constraints from other high-mass resonance searches made at the $8-\mathrm{TeV}$ LHC. This model was originally proposed to explain the excess at around $2 \mathrm{TeV}$ in the searches for a diboson resonance in the ATLAS experiment [26]. As we will see, we can readily explain the $750-\mathrm{GeV}$ resonance by lowering the dynamical scale and mass parameters in the model.

In this model, the scalar resonance appears as the lightest composite state under hidden strong dynamics at around the TeV scale. A peculiar feature of the model is that the hidden sector consists of scalar quarks, and the lightest composite state is not a pseudoGoldstone boson. With this feature, the mass of the resonance should be in close proximity to the dynamical scale, unlike in the models where the resonance is identified with a pseudo Goldstone modes. As a result, the model predicts intriguing signatures associated with the hidden dynamics at the LHC such as leptons, jets and leptons with multiple photons as well as the existence of charged composite resonances in companion with the $750-\mathrm{GeV}$ resonance. We also associate the $\mathrm{TeV}$-scale dynamics behind the resonance with an explanation of dark matter. 


\section{A scalar resonance from hidden dynamics}

In the model of ref. [25], the scalar resonance, $S$ with mass $M_{S}$, couples to the gauge bosons in the Standard Model (SM) due to the SM gauge charges of the constituent hidden scalar quarks. Such interactions are described by the effective Lagrangian (see also ref. [27] for earlier works):

$$
\mathcal{L}_{\text {eff }}=\frac{1}{\Lambda_{3}} S G_{\mu \nu}^{a} G^{a \mu \nu}+\frac{1}{\Lambda_{2}} S W_{\mu \nu}^{i} W^{i \mu \nu}+\frac{5}{3} \frac{1}{\Lambda_{1}} S B_{\mu \nu} B^{\mu \nu},
$$

where, $\Lambda_{1,2,3}$ are suppression scales which are related to the dynamical scale of the hidden sector, and $G, W$ and $B$ are the field strengths of the $\mathrm{SU}(3)_{C}, \mathrm{SU}(2)_{L}$, and $\mathrm{U}(1)_{Y}$ gauge bosons, respectively. These gauge fields are normalized so that their kinetic terms are given by

$$
\mathcal{L}=-\frac{1}{4 g_{s}^{2}} G_{\mu \nu}^{a} G^{a \mu \nu}-\frac{1}{4 g^{2}} W_{\mu \nu}^{i} W^{i \mu \nu}-\frac{1}{4 g^{\prime 2}} B_{\mu \nu} B^{\mu \nu},
$$

with $g_{s}, g$ and $g^{\prime}$ being the corresponding gauge coupling constants, and the superscripts $a$ and $i$ denoting the indices for the corresponding adjoint representations.

Through the effective interaction with the gluons and in the narrow width approximation, the scalar resonance is produced at the LHC via the gluon fusion process

$$
\begin{aligned}
\sigma(p+p \rightarrow S) & =\frac{\pi^{2}}{8}\left(\frac{\Gamma(S \rightarrow g+g)}{M_{S}}\right) \times\left[\frac{1}{s} \frac{\partial \mathcal{L}_{g g}}{\partial \tau}\right], \\
\frac{\partial \mathcal{L}_{g g}}{\partial \tau} & =\int_{0} d x_{1} d x_{2} f_{g}\left(x_{1}\right) f_{g}\left(x_{2}\right) \delta\left(x_{1} x_{2}-\tau\right),
\end{aligned}
$$

where $\tau=M_{S}^{2} / s$ and $\sqrt{s}$ denotes the center-of-mass energy of the proton-proton collision. Using the of MSTW2008 parton distribution functions (PDF's) [28], we obtain

$$
\frac{1}{s} \frac{\partial \mathcal{L}_{g g}}{\partial \tau} \simeq \begin{cases}0.97 \times 10^{3} \mathrm{pb}(\text { for } \sqrt{s}=8 \mathrm{TeV}), \\ 4.4 \times 10^{3} \mathrm{pb} \quad(\text { for } \sqrt{s}=13 \mathrm{TeV}),\end{cases}
$$

where we fixed the factorization scale and the renormalization scale at $\mu=M_{S} / 2$ for $M_{S} \simeq 750 \mathrm{TeV} .{ }^{1}$

The partial decay widths of the scalar resonance are given by

$$
\begin{aligned}
\Gamma(S \rightarrow g+g) & =\frac{2}{\pi}\left(\frac{g_{s}^{2}}{\Lambda_{3}}\right)^{2} M_{S}^{3}, \\
\Gamma\left(S \rightarrow W^{+}+W^{-}\right) & =\frac{1}{2} \frac{1}{\pi}\left(\frac{g^{2}}{\Lambda_{2}}\right)^{2} M_{S}^{3}, \\
\Gamma(S \rightarrow Z+Z) & =\frac{1}{4} \frac{1}{\pi}\left[\left(\frac{g^{2}}{\Lambda_{2}}\right) c_{W}^{2}+\frac{5}{3}\left(\frac{g^{\prime 2}}{\Lambda_{1}}\right) s_{W}^{2}\right]^{2} M_{S}^{3}, \\
\Gamma(S \rightarrow \gamma+\gamma) & =\frac{1}{4} \frac{1}{\pi}\left[\left(\frac{g^{2}}{\Lambda_{2}}\right) s_{W}^{2}+\frac{5}{3}\left(\frac{g^{\prime 2}}{\Lambda_{1}}\right) c_{W}^{2}\right]^{2} M_{S}^{3}, \\
\Gamma(S \rightarrow Z+\gamma) & =\frac{1}{2} \frac{1}{\pi}\left[\left(\frac{g^{2}}{\Lambda_{2}}\right)-\frac{5}{3}\left(\frac{g^{\prime 2}}{\Lambda_{1}}\right)\right]^{2} c_{W}^{2} s_{W}^{2} M_{S}^{3},
\end{aligned}
$$

\footnotetext{
${ }^{1}$ See e.g., ref. [29] for a discussion on higher-order QCD corrections, i.e., the $K$-factor, for the production of the scalar resonance.
} 


\begin{tabular}{|ccccc|}
\hline & $\mathrm{SU}(5)$ & $\mathrm{SU}(3)_{C}$ & $\mathrm{SU}(2)_{L}$ & $\mathrm{U}(1)_{Y}$ \\
\hline$Q_{L}$ & $\overline{\mathbf{5}}$ & $\mathbf{1}$ & $\mathbf{2}$ & $1 / 2$ \\
$Q_{D}$ & $\overline{\mathbf{5}}$ & $\overline{\mathbf{3}}$ & $\mathbf{1}$ & $-1 / 3$ \\
\hline
\end{tabular}

Table 1. Charge assignments of the bi-fundamental scalars under the hidden SU(5) and the SM gauge symmetries. The SM gauge charges of the $Q$ 's are assigned so that they form an antifundamental representation of $\mathrm{SU}(5)_{\mathrm{GUT}}$.

where $s_{W} \equiv \sin \theta_{W}$ and $c_{W}=\left(1-s_{W}^{2}\right)^{1 / 2}$ with $\theta_{W}$ being the weak mixing angle. The masses of the $W$ and $Z$ bosons are neglected to a good approximation.

Now let us discuss the model content and hidden dynamics that lead to the scalar resonance. Following ref. [25], we consider a set of scalar fields $Q$ 's that carry both the hidden $\mathrm{SU}\left(N_{h}\right)$ and the $\mathrm{SM}$ gauge charges. The $\mathrm{SU}\left(N_{h}\right)$ interaction is assumed to become strong at a dynamical scale $\Lambda_{\text {dyn }}$. Explicitly, we take $N_{h}=5$. The charge assignments of $Q$ 's are given in table 1. It should be noted that we assign the SM gauge charges to $Q$ 's in such a way that they form an anti-fundamental representation of the minimal SU(5) grand unified theory (GUT).

The bi-fundamental scalars are assumed to have masses, ${ }^{2} m_{D, L}$ :

$$
\mathcal{L} \supset-m_{D}^{2} Q_{D}^{\dagger} Q_{D}-m_{L}^{2} Q_{L}^{\dagger} Q_{L}
$$

When the masses of the scalar quarks do not exceed $\Lambda_{\mathrm{dyn}}$, the lightest composite state is expected to be a $C P$-even neutral composite scalar that is a mixture of $Q_{L}^{\dagger} Q_{L}, Q_{D}^{\dagger} Q_{D}$, and a $C P$-even glueball. It should be emphasized here that the lightest neutral scalar is expected to be lighter than the other SM-charged composite states due to mixing, since the charged scalar composite fields are not accompanied by mixing partners. This situation should be compared with models with fermionic bi-fundamental representations where the lightest singlet appears as a Goldstone boson mode. In this case, one of the neutral Goldstone bosons becomes heavier than the SM-charged Goldstone bosons due to the chiral anomaly of the hidden gauge interaction. Thus, if we further take the mass parameter of the colored hidden quark, $m_{D}$, larger than $\Lambda_{\text {dyn }}$, no neutral Goldstone boson remains lighter than the SM-charged ones, such as the $\mathrm{SU}(2)_{L}$ triplet Goldstone bosons. In our scalar quark model, on the other hand, we expect that the neutral scalar boson remains lighter than the SM-charged composite bosons even if we take $m_{D}$ larger than $\Lambda_{\text {dyn }}$ due to the mixing with the glueball. This feature may be important when we discuss the phenomenology of the charged composite states (see discussions at the end of this section).

Before proceeding further, let us discuss the vacuum structure of the scalar quark model more closely. For that purpose, it is most transparent to discuss in the Higgs phase of this model. In fact, there is strong evidence that (lattice) gauge theories with scalars in the fundamental representations have no sharp phase boundaries which separate the confined phase and the Higgs phase [30-32]. Thus, by deciphering the vacuum structure

\footnotetext{
${ }^{2}$ The bi-fundamental scalars also possess quartic couplings although they are not relevant for the later discussion.
} 
in the Higgs phase, it is possible to infer the vacuum structure in the confined phase. To discuss the Higgs phase of this model, let us assume that $m_{D, L}^{2}$ in eq. (2.10) are negative and their sizes are larger than $\Lambda_{\mathrm{dyn}}^{2}$. For such parameters, we find that the bi-fundamental scalars, $Q_{D, L}$, develop a condensate and break the symmetries. Importantly, however, the diagonal subgroups $\mathrm{SU}(3) \times \mathrm{SU}(2) \times \mathrm{U}(1)$, playing the role of $\mathrm{SM}$ gauge symmetries, remain unbroken in the Higgs phase. This strongly indicates that they also do so in the confined phase when $m_{D, L} \ll \Lambda_{\text {dyn }}$. Armed with this argument, we assume that the SM gauge symmetries are not broken in the confined phase.

In our analysis, we are most interested in how the singlet $S$ couples to the SM gauge bosons. For this purpose, we parametrise the relative contributions of $\left[Q_{L}^{\dagger} Q_{L}\right]$ and $\left[Q_{D}^{\dagger} Q_{D}\right]$ by a mixing parameter $\theta_{Q}$ :

$$
S \propto \cos \theta_{Q} \times\left[Q_{L}^{\dagger} Q_{L}\right]+\sin \theta_{Q} \times\left[Q_{D}^{\dagger} Q_{D}\right] .
$$

For example, the $Q_{D}^{\dagger} Q_{D}$ content is expected to be suppressed for $m_{D} \gg m_{L}$. A quantitative estimation of $\theta_{Q}$ is, however, difficult due to the non-perturbative nature of the strong interaction. Hence we take $\theta_{Q}$ as a free parameter in the following analysis. ${ }^{3}$ For $m_{D} \gtrsim$ $\Lambda_{\text {dyn }}$, the second contribution can be effectively regarded as the glueball contribution that couples to the gauge bosons through the $Q_{D}$-loop diagrams (see also ref. [33]).

To match the scalar resonance in the effective field theory onto the composite states, we rely on the Naive Dimensional Analysis (NDA) [34, 35], leading to

$$
S \simeq \frac{4 \pi}{\kappa \Lambda_{\mathrm{dyn}}} \cos \theta_{Q} \times\left[Q_{L}^{\dagger} Q_{L}\right]+\frac{4 \pi}{\kappa \Lambda_{\mathrm{dyn}}} \sin \theta_{Q} \times\left[Q_{D}^{\dagger} Q_{D}\right]
$$

with a canonical kinetic term. The parameter $\kappa$ represents $\mathcal{O}(1)$ uncertainties of the NDA. Altogether, we obtain the effective interactions of $S$ to the SM gauge bosons as

$$
\begin{aligned}
\mathcal{L}_{\text {eff }}= & \frac{\kappa}{4 \pi \Lambda_{\mathrm{dyn}}} \sin \theta_{Q} S G_{\mu \nu}^{a} G^{a \mu \nu}+\frac{\kappa}{4 \pi \Lambda_{\mathrm{dyn}}} \cos \theta_{Q} S W_{\mu \nu}^{i} W^{i \mu \nu} \\
& +\frac{2 \kappa}{4 \pi \Lambda_{\mathrm{dyn}}}\left(\frac{\sin \theta_{Q}}{3}+\frac{\cos \theta_{Q}}{2}\right) S B_{\mu \nu} B^{\mu \nu} .
\end{aligned}
$$

As a result, the coefficients in the effective interactions given in eq. (2.1) are given by

$$
\frac{1}{\Lambda_{3}}=\frac{\kappa \sin \theta_{Q}}{4 \pi \Lambda_{\mathrm{dyn}}}, \frac{1}{\Lambda_{2}}=\frac{\kappa \cos \theta_{Q}}{4 \pi \Lambda_{\mathrm{dyn}}}, \frac{1}{\Lambda_{1}}=\frac{\kappa}{4 \pi \Lambda_{\mathrm{dyn}}} \frac{6}{5}\left(\frac{\sin \theta_{Q}}{3}+\frac{\cos \theta_{Q}}{2}\right),
$$

Therefore, the production rates and the branching ratios are determined by two parameters, $\sin \theta_{Q}$ and $\Lambda_{\mathrm{dyn}}$, in this model.

Figure 1 shows the branching ratios of the scalar resonance as functions of $\sin \theta_{Q}$ for $M_{S}=750 \mathrm{GeV}$ and $\Lambda_{\mathrm{dyn}}=1 \mathrm{TeV}$. Here we use the running gauge coupling constants at the renormalization scale $M_{S}$. The plot shows that the branching ratios of the $W W$ and $Z Z$ modes are about nine and three times larger than that of the $\gamma \gamma$ modes for most of the parameter region. On the other hand, the branching ratio into gluons is suppressed compared to even that of $\gamma \gamma$ for small $\sin \theta_{Q}$, as is evident from eq. (2.14).

\footnotetext{
${ }^{3}$ We assume that the hidden strong dynamics does not cause spontaneous breaking of the SM gauge symmetries, although $\left\langle Q^{\dagger} Q\right\rangle$ is expected to be non-vanishing. In particular, the mass terms of $Q$ 's lead to linear terms of the SM singlet composite scalars, resulting in $\left\langle Q^{\dagger} Q\right\rangle \neq 0$.
} 


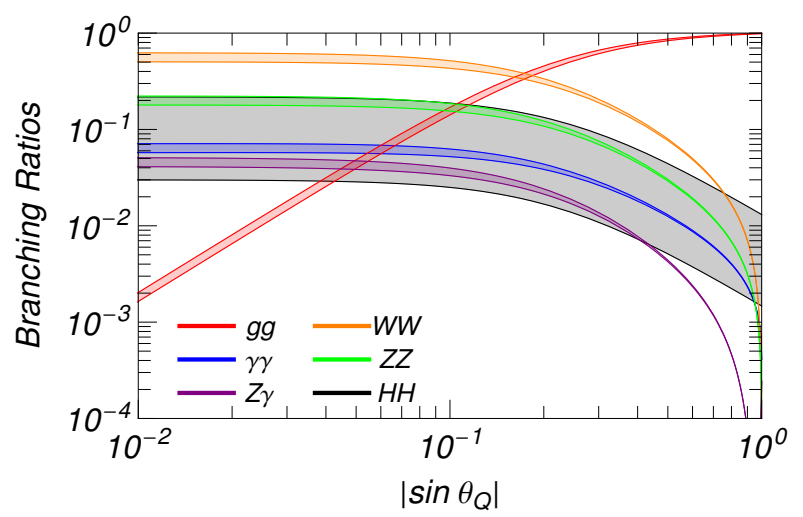

Figure 1. Branching ratios of the scalar resonance into the gauge boson pairs and the Higgs boson pair as functions of $\sin \theta_{Q}$ for $M_{S}=750 \mathrm{GeV}$ and $\Lambda_{\mathrm{dyn}}=1 \mathrm{TeV}$. The colored bands indicate the ranges of predictions as $\lambda$ is varied from 0.1 to 0.3 .

In the figure, we also take into account the decay of $S$ into a pair of the $125-\mathrm{GeV}$ Higgs bosons due to interactions between $Q$ 's and Higgs boson $H$,

$$
\mathcal{L}=\lambda_{L, D} Q_{L, D}^{\dagger} Q_{L, D} H^{\dagger} H
$$

with $\lambda_{L, D}$ being coupling constants. These interactions induce an effective interaction between $S$ and Higgs doublets,

$$
\mathcal{L} \sim \frac{\lambda}{4 \pi} \Lambda_{\mathrm{dyn}} S H^{\dagger} H+\lambda S^{2} H^{\dagger} H+\cdots,
$$

where we again use the NDA and reparameterize $\lambda_{L, D}$ and $\theta_{Q}$ by $\lambda$. Through this operator, the resonance decays into a pair of Higgs bosons with a partial decay width: ${ }^{4}$

$$
\Gamma\left(S \rightarrow H+H^{\dagger}\right)=\frac{1}{8 \pi M_{S}}\left(\frac{\lambda \Lambda_{\mathrm{dyn}}}{4 \pi}\right)^{2} .
$$

In figure 1 , we show the branching ratio of this mode for $\lambda=0.1-0.3$. We also show how the branching ratios into the gauge bosons are affected by the the Higgs pair mode as colored bands. As is shown, the branching ratio into the Higgs bosons, proportional to $\lambda^{2}$, is subdominant for most of the parameter region. Thus, its effects on the branching ratios of the modes of gauge boson pairs are not significant, as indicated by the narrow bands, and become diminishing when $\lambda$ is much smaller than 0.1 .

In the following, we discuss the preferred parameter region to explain the diphoton excess at $750 \mathrm{GeV}$ while being consistent with all the constraints from the searches for the other modes of gauge boson pairs. ${ }^{5}$ In view of this, we simply neglect the effects of the operator in eq. (2.16) by assuming $\lambda \lesssim 0.3 .^{6}$

\footnotetext{
${ }^{4}$ Strictly speaking, the operator in eq. (2.16) also induces the decays into the weak gauge bosons.

${ }^{5}$ The searches for a resonance decaying into a pair of Higgs bosons have imposed an upper bound of $\sigma(p p \rightarrow S \rightarrow h h) \lesssim 39 \mathrm{fb}$ [36], which can be satisfied in most of the parameter region in figure 2 .

${ }^{6}$ As discussed in ref. [25], a similar quartic coupling between the $\mathrm{SU}(2)_{L}$ composite triplet scalar and a pair of Higgs doublets leads to a non-vanishing vacuum expectation value of the composite triplet scalar. Due to electroweak precision constraints, the typical size of the quartic couplings should be at most $\mathcal{O}(0.1)$ for the model to be successful.
} 
Several comments are in order. The singlet and the Higgs boson mix through the terms in eq. (2.16), leading to a mixing angle

$$
\varepsilon \sim \frac{\lambda v \Lambda}{4 \pi M_{S}^{2}}
$$

where $v$ denotes the vacuum expectation value of the SM Higgs doublet, i.e., $v \simeq 174 \mathrm{GeV}$. Such a mixing of the Higgs boson is constrained by the measurements of the observed 125$\mathrm{GeV}$ Higgs coupling strengths at the LHC as well as the electroweak precision data. We find that those constraints can be satisfied for $\Lambda_{\text {dyn }}=O(1) \mathrm{TeV}$ and $M_{S} \simeq 750 \mathrm{GeV}$ as long as $\lambda<\mathcal{O}(1)$ (see, e.g., refs. [47, 48]). It should be also noted that $S$ is expected to acquire a vacuum expectation value of $\mathcal{O}\left(\Lambda_{\mathrm{dyn}}\right) / 4 \pi$ in the NDA. Accordingly, the Higgs mass receives a contribution from the condensation of $S$ through eq. (2.16) of about $\lambda \times \mathcal{O}\left(100^{2}\right) \mathrm{GeV}^{2}$. Again, such a contribution is not very significant as long as $\lambda<\mathcal{O}(1)$.

Now, let us discuss the favored parameter region on the $\left(\sin \theta_{Q}, \Lambda_{\text {dyn }}\right)$ plane. Figure 2 shows in blue curves the contours of the cross section of the diphoton signal at the $13-\mathrm{TeV}$ LHC. The colored regions are excluded by the resonance searches using various decays into gauge bosons for $M_{S}=750 \mathrm{GeV}$ at the 8 - TeV LHC. Here, we use the compilation of constraints listed in ref. $[5] ;^{7}$

$$
\begin{array}{rlrl}
\sigma(p p \rightarrow S \rightarrow \gamma \gamma) & <1.5 \mathrm{fb} & & {[37,38]} \\
\sigma(p p \rightarrow S \rightarrow W W) & <40 \mathrm{fb} & & {[39,40]} \\
\sigma(p p \rightarrow S \rightarrow Z Z) & <12 \mathrm{fb} & & {[41],} \\
\sigma(p p \rightarrow S \rightarrow Z \gamma) & <11 \mathrm{fb} & & {[42],} \\
\sigma(p p \rightarrow S \rightarrow j j) & <2.5 \mathrm{pb} & {[43,44]}
\end{array}
$$

The figure shows that the model can successfully explain the diphoton excess, $\sigma_{\gamma \gamma}^{(13 \mathrm{TeV})}=$ $\mathcal{O}(1) \mathrm{fb}$ while evading all the above-mentioned constraints for

$$
\Lambda_{\text {dyn }} \simeq(10 \mathrm{TeV}-30 \mathrm{TeV}) \times \sin \theta_{Q} .
$$

Since the composite scalar mass is expected to be at around $\Lambda_{\mathrm{dyn}}$, we find that an appropriate range of the mixing angle is $\sin \theta_{Q} \simeq 10^{-1}-10^{-1.5}$. This result also implies that the mass parameter $m_{D}$ is larger than $m_{L}$ and $\Lambda_{\text {dyn }}$. Moreover, $\Gamma_{S} / M_{S} \sim \mathcal{O}\left(10^{-4}\right)$, justifying our narrow width approximation. ${ }^{8}$

Let us also comment on the production cross sections of the other gauge boson modes. In the favored parameter region, $\sin \theta_{Q} \simeq 10^{-1}-10^{-1.5}$, the branching ratios of the $W W$, $Z Z, Z \gamma$ modes are almost constant as a function of $\sin \theta_{Q}$, while that of the $g g$ modes

\footnotetext{
${ }^{7}$ The constraints on $\sigma(p p \rightarrow S \rightarrow Z \gamma)$ is weaker than other constraints, and hence we do not show it in figure 2.

${ }^{8}$ The ATLAS Collaboration seems to suggest a sizeable width of the resonance, $\Gamma_{S} / M_{S} \sim 6 \%$. In our model, however, such a large width requires a very small dynamical scale $\Lambda_{\text {dyn }} \lesssim 100 \mathrm{GeV}$, which leads to too light composite states. Thus, to achieve a large width, one needs to extend the model so that the lightest composite particles decay into some new neutral particles.
} 


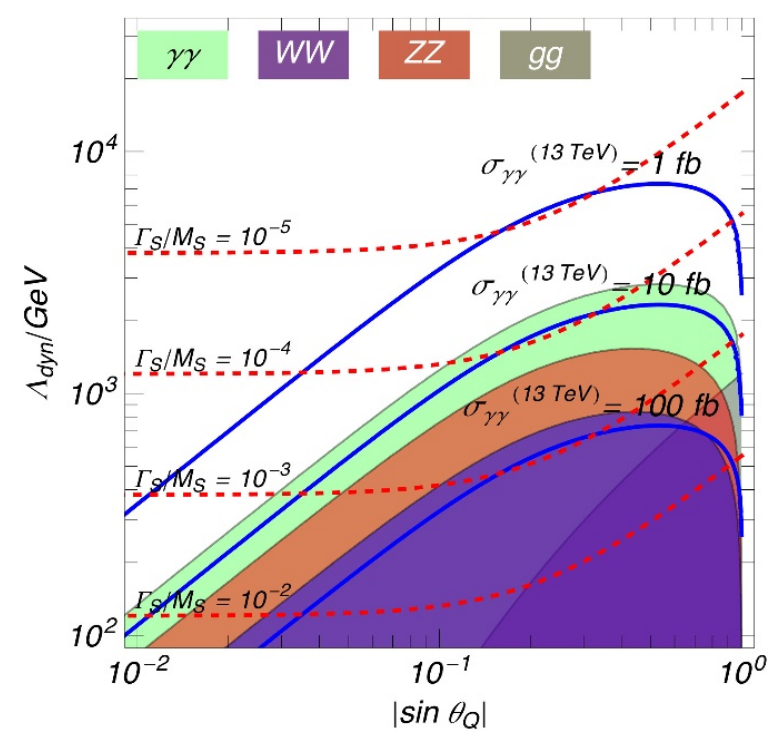

Figure 2. Contours of the production cross section of $S$ times its decay branching ratio into $\gamma \gamma$ at the $13-\mathrm{TeV}$ LHC by assuming the gluon fusion production process. We fix $M_{S}=750 \mathrm{GeV}$ and take the factorization and renormalization scales at $\mu=M_{S} / 2$. The color-shaded regions are excluded by the searches through various decay modes in LHC Run I as detailed in the main text. The dashed red curves show the narrowness of the decay width.

simply scales by $\sin ^{2} \theta_{Q}$. Thus, the the production cross sections of the other modes are predicted to be

$$
\begin{aligned}
\sigma(p p \rightarrow S \rightarrow W W) & \simeq 9 \times \sigma(p p \rightarrow S \rightarrow \gamma \gamma), \\
\sigma(p p \rightarrow S \rightarrow Z Z) & \simeq 3 \times \sigma(p p \rightarrow S \rightarrow \gamma \gamma), \\
\sigma(p p \rightarrow S \rightarrow Z \gamma) & \simeq 0.7 \times \sigma(p p \rightarrow S \rightarrow \gamma \gamma), \\
\sigma(p p \rightarrow S \rightarrow j j) & \simeq 270 \sin ^{2} \theta_{Q} \times \sigma(p p \rightarrow S \rightarrow \gamma \gamma),
\end{aligned}
$$

respectively, which will be tested by the LHC Run-II experiments. ${ }^{9}$

Before closing this section, let us comment on the SM-charged composite states predicted in this model. Since the hidden sector consists of $Q_{D}$ and $Q_{L}$, the model predicts not only the singlet composite scalar, but also the charged composites: an $\mathrm{SU}(3)_{C}$ octet, an $\mathrm{SU}(2)_{L}$ triplet, and a bi-fundamental representation of $\mathrm{SU}(3)_{C} \times \mathrm{SU}(2)_{L}$ with a hypercharge of $5 / 6$.

Due to the color charge of the octet scalar, it is directly produced through the $\mathrm{SU}(3)_{C}$ gauge interaction at the LHC and decays into a pair of gluons. By the searches at the 8-TeV LHC, the production cross section of the octet scalar with a mass around $1 \mathrm{TeV}$ is constrained to be around $\mathcal{O}(1) \mathrm{pb}[43,45]$, which is much larger than the pair production cross section of the octet scalars [46] as well as the single production rate via eq. (2.1). It

\footnotetext{
${ }^{9}$ If the coupling to the Higgs doublet in eq. (2.16) is sizeable, the above predictions can be slightly modified.
} 
should be noted that the octet scalar mass is expected to be larger than that of $S$ because $S$ is the lightest admixture of $\left[Q_{L}^{\dagger} Q_{L}\right]$ and $\left[Q_{D}^{\dagger} Q_{D}\right]$ while the octet is a unique scalar state.

Similarly, the triplet scalar is produced via the Drell-Yan process and immediately decays into SM electroweak gauge bosons and Higgs bosons through the interaction in eq. (2.16). Unlike the neutral scalar $S$, the triplet scalar does not couple to the gluons via dimension- 5 operators. To date, there is no stringent constraint on the triplet scalar with a mass of $\mathcal{O}(1) \mathrm{TeV}$.

The scalar in the bi-fundamental representation of $\mathrm{SU}(3)_{C} \times \mathrm{SU}(2)_{L}$ requires special care, as it cannot decay into a pair of SM gauge bosons due to its charges. To make it decay promptly, we introduce one flavor of fermions under the hidden $\mathrm{SU}(5)$ gauge symmetry $\left(\psi_{Q}, \bar{\psi}_{Q}\right)$, which allow $Q$ 's to couple to the SM quarks and leptons, $\bar{d}_{R}$ and $\ell_{L}$, via

$$
\mathcal{L} \supset y Q_{D}^{\dagger} \psi_{Q} \bar{d}_{R}+y Q_{L}^{\dagger} \psi_{Q} \ell_{L}+M_{Q} \psi_{Q} \bar{\psi}_{Q}+\text { h.c. }
$$

Here, $y$ denotes a coupling constant and $M_{Q}$ the mass of the fermion $\psi_{Q}$. We take $M_{Q}$ to be much larger than a TeV, so that they are not produced at the LHC.

Through these interactions, the $\left[Q_{D}^{\dagger} Q_{L}\right]$ bound states decay into $\bar{d}_{R}^{\dagger}+\ell_{L}+S,{ }^{10}$ which is estimated to be roughly

$$
\Gamma \sim\left(\frac{1}{16 \pi}\right)^{2}\left(\frac{y^{2}}{4 \pi}\right)^{2} \frac{\Lambda_{\mathrm{dyn}}^{2}}{M_{Q}^{4}} M_{\left[Q_{D}^{\dagger} Q_{L}\right]}^{3},
$$

where $M_{\left[Q_{D}^{\dagger} Q_{L}\right]}$ denotes the mass of the bound state. For $M_{Q} \lesssim 10^{4} \mathrm{GeV}$, the bound state decays promptly into down-type quarks and leptons and $S$ which subsequently decays into jets, $W W, Z Z, Z \gamma$ or $\gamma \gamma$ as discussed before.

It should be noted that the new interactions in eq. (2.25) lead to flavor-changing neutral-current interactions of $d_{R}^{4}$ suppressed by a loop factor $y^{4} /\left(16 \pi^{2} M_{Q}^{2}\right)$. Thus, to evade the constraints, it is safe to assume that the couplings in eq. (2.25) are sufficiently small, $y \lesssim 10^{-2}$ for $M_{Q} \simeq 10 \mathrm{TeV}$ (see, e.g., ref. [57]), with which the bi-fundamental composites could leave a measurable decay length.

For a larger $M_{Q}$, e.g., $M_{Q} \gtrsim 10^{7} \mathrm{GeV}$, the bound state can be stable within the detectors and give a striking signature. The lower mass limit put by the results of searches for heavy stable charged particles at CMS ranges up to $0.9-1 \mathrm{TeV}$, depending on the QED charges [49]. ${ }^{11}$ It should also be noted that for $M_{Q} \gg 10^{8}-10^{9} \mathrm{GeV}$, the lifetime of the bound state becomes longer than $\mathcal{O}(1)$ second and spoils the success of the Big Bang Nucleosynthesis [52-54]. ${ }^{12}$

\section{Discussions and conclusions}

In this paper, we have revisited a model of scalar composite resonance that couples to the SM gauge bosons via the higher-dimensional operators proposed in ref. [25] in light of the

\footnotetext{
${ }^{10}$ The two-body decay width into $\bar{d}_{R}^{\dagger}$ and $\ell_{L}$ is suppressed by the masses of the fermions.

${ }^{11}$ When the mass of the scalar $\left[Q_{D}^{\dagger} Q_{L}\right]$ bound state is $1 \mathrm{TeV}$, the production cross section is $0.2 \mathrm{fb}$ at $8 \mathrm{TeV}[50]$ and $6 \mathrm{fb}$ at $13 \mathrm{TeV}[51]$.

${ }^{12}$ See also ref. [56] for related discussions.
} 
750-GeV diphoton excess discovered recently in LHC Run-II. In this model, the lightest composite state is expected to be the $C P$-even singlet scalar which is the admixture of the neutral bi-linear composite of the scalar quarks and a glueball. As we have shown, the model can consistently explain the excess while evading all the constraints from other highmass resonance searches made in LHC Run-I. It should be noted that the $C P$ property of the resonance can be tested by measuring the angular distribution of the four leptons in the final states of the $Z Z$ modes (see, e.g., ref. [57]). Thus, this composite scenario can be clearly distinguished from the other composite models where the neutral scalar manifests as a $C P$-odd pseudo-Goldstone mode.

The neutral scalar boson is accompanied by many charged bound states whose masses are also in the $\mathrm{TeV}$ regime. Therefore, we expect that the LHC Run-II experiments will discover a zoo of such particles around that scale. In particular, the bound state of $\left[Q_{L}^{\dagger} Q_{D}\right]$ has a striking signature of decaying into a lepton, a down-type quark and $S$, or it can even leave charged tracks inside the detector when the bound state is sufficiently stable.

As a peculiar feature of this model, the mass of the lightest composite state is not separable from the dynamical scale of the hidden sector, as it is not protected for any symmetry reasons. Thus, the dynamical scale should be in close proximity to the composite mass, unlike again the models in which the $750-\mathrm{GeV}$ resonance is identified as a pseudoGoldstone boson. Therefore, we expect that the quark-like picture of the hidden sector emerges at a rather low energy in future collider experiments. For example, production of multiple partons in the hidden sector becomes possible and ends up with events of multiple jets, multiple leptons and multiple photons.

Before closing this paper, let us address an important question: "who ordered the 750GeV resonance?" One ambitious answer is the dark matter candidate. In fact, as discussed in ref. [25], this model has a good dark matter candidate: the lightest baryonic scalar

$$
B \propto Q Q Q Q Q
$$

This state is neutral under the SM gauge group due to the choice of $N_{h}=5 .{ }^{13}$ It should be emphasized that the neutralness of the lightest baryonic state under the SM gauge group is one of the prominent features of this model. If, instead, the hidden sector consists of bi-fundamental fermions, the neutral baryonic state is expected to be heavier than the lightest but SM-charged baryonic state since the neutral baryonic state has a larger orbital angular momentum inside.

In the early universe, the baryonic scalars annihilate into a pair of lighter scalar nonbaryonic composite states. The thermal relic abundance would be much lower than the observed dark matter density if the annihilation cross section (into $S$, glueballs, etc.) saturates the unitarity limit [58]. The abundance of $B$ is roughly given by

$$
\Omega_{B} h^{2} \sim 0.25 \times 10^{-3} \frac{1}{F\left(M_{B}\right)^{4}}\left(\frac{M_{B}}{5 \mathrm{TeV}}\right)^{2}
$$

\footnotetext{
${ }^{13}$ To make $B$ stable, a (discrete) symmetry is required. We presume that such a symmetry is not broken spontaneously by the strong dynamics as long as $m_{D, L}^{2} \neq 0$.
} 
where $M_{B}$ is the mass of $B$ and $F\left(M_{B}\right)$ denotes the form factor of the interactions of $B$ with the lighter states. ${ }^{14}$ By remembering $M_{B} \gg \Lambda_{\text {dyn }}$ (in particular when $m_{D} \gg \Lambda_{\text {dyn }}$ ), it is expected that the form factor is slightly smaller than 1 . Therefore, the thermal relic abundance of $B$ can be consistent with the observed dark matter density, although a quantitative estimation is difficult due to our inability to estimate the form factor precisely.

Finally, let us comment on the direct detection of the dark matter candidate. The coupling between $Q$ 's and the Higgs doublet in eq. (2.16) also leads to a direct coupling between the scalar dark matter and the Higgs doublet,

$$
\mathcal{L}=\lambda_{B} B^{\dagger} B H^{\dagger} H
$$

where $\lambda_{B}$ is of $\mathcal{O}\left(\lambda_{L, D}\right) .{ }^{15}$ Thus, the dark matter interacts elastically with nuclei via the Higgs boson exchange, resulting in a spin-independent cross section [59]

$$
\sigma_{S I}=\frac{\lambda_{B}^{2}}{4 \pi m_{h}^{4}} \frac{m_{N}^{4} f_{N}^{2}}{M_{B}^{2}} \simeq 5.4 \times 10^{-46} \mathrm{~cm}^{2} \times \lambda_{B}^{2}\left(\frac{5 \mathrm{TeV}}{M_{B}}\right)^{2},
$$

where we have used the lattice result $f_{N} \simeq 0.326$ [60]. Although this is much smaller than the current limit $\sigma_{S I} \lesssim 5 \times 10^{-44} \mathrm{~cm}^{2}\left(M_{B} / 5 \mathrm{TeV}\right)$ by the LUX experiment [61], it is within the reach of the proposed LUX-Zeplin (LZ) experiment [62], with details depending on the coupling constants and the dark matter mass.

Note added. During the reviewing process of the manuscript, the ATLAS and CMS Collaborations updated their diphoton analyses at the Moriond 2016 conference [63, 64] with global fits of signal rates given by (see, for example, ref. [65]):

$$
\begin{aligned}
& \sigma(p p \rightarrow S) \times B r(S \rightarrow \gamma \gamma)=(5.5 \pm 1.5) \mathrm{fb}, \quad(\text { ATLAS }), \\
& \sigma(p p \rightarrow S) \times B r(S \rightarrow \gamma \gamma)=(4.8 \pm 2.1) \mathrm{fb}, \quad(\mathrm{CMS}),
\end{aligned}
$$

which are in better agreement with the $8-\mathrm{TeV}$ result. Moreover, the local statistical significance of the excess around $750 \mathrm{GeV}$ went up to $3.9 \sigma$ in ATLAS and $3.4 \sigma$ in CMS.

\section{Acknowledgments}

This work is supported in part by the Ministry of Science and Technology of Taiwan under Grant No. MOST104-2628-M-008-004-MY4 (C.-W. C); Grants-in-Aid for Scientific Research from the Ministry of Education, Culture, Sports, Science, and Technology (MEXT), Japan, No. 24740151 and No. 25105011 (M. I.) as well as No. 26104009 (T. T. Y.); Grantin-Aid No. 26287039 (M. I. and T. T. Y.) from the Japan Society for the Promotion of Science (JSPS); and by the World Premier International Research Center Initiative (WPI), MEXT, Japan (M. I., and T. T. Y.). MI is grateful to the Center for Mathematics and Theoretical Physics and Department of Physics of National Central University for hospitality where this work is completed.

\footnotetext{
${ }^{14}$ We define the form factor in such a way that the interaction of $B$ saturates the unitarity limit when $F=1$.

${ }^{15}$ The dark matter also annihilates into Higgs bosons via this interactions, although its annihilation cross section is subdominant for $\lambda_{B}=\mathcal{O}(0.1)$ [59].
} 
Open Access. This article is distributed under the terms of the Creative Commons Attribution License (CC-BY 4.0), which permits any use, distribution and reproduction in any medium, provided the original author(s) and source are credited.

\section{References}

[1] ATLAS collaboration, Search for resonances decaying to photon pairs in 3.2 $\mathrm{fb}^{-1}$ of $\mathrm{pp}$ collisions at $\sqrt{s}=13 \mathrm{TeV}$ with the ATLAS detector, ATLAS-CONF-2015-081 (2015).

[2] CMS collaboration, Search for new physics in high mass diphoton events in proton-proton collisions at $\sqrt{s}=13 \mathrm{TeV}$, CMS-PAS-EXO-15-004 (2015).

[3] D. Buttazzo, A. Greljo and D. Marzocca, Knocking on new physics' door with a scalar resonance, Eur. Phys. J. C 76 (2016) 116 [arXiv:1512.04929] [INSPIRE].

[4] S. Knapen, T. Melia, M. Papucci and K. Zurek, Rays of light from the LHC, Phys. Rev. D 93 (2016) 075020 [arXiv: 1512.04928] [INSPIRE].

[5] R. Franceschini et al., What is the $\gamma \gamma$ resonance at 750 GeV?, JHEP 03 (2016) 144 [arXiv: 1512.04933] [INSPIRE].

[6] S.D. McDermott, P. Meade and H. Ramani, Singlet Scalar Resonances and the Diphoton Excess, Phys. Lett. B 755 (2016) 353 [arXiv:1512.05326] [INSPIRE].

[7] J. Ellis, S.A.R. Ellis, J. Quevillon, V. Sanz and T. You, On the Interpretation of a Possible $\sim 750 \mathrm{GeV}$ Particle Decaying into $\gamma \gamma$, JHEP 03 (2016) 176 [arXiv:1512.05327] [INSPIRE].

[8] M. Low, A. Tesi and L.-T. Wang, A pseudoscalar decaying to photon pairs in the early LHC Run 2 data, JHEP 03 (2016) 108 [arXiv:1512.05328] [INSPIRE].

[9] R.S. Gupta, S. Jäger, Y. Kats, G. Perez and E. Stamou, Interpreting a $750 \mathrm{GeV}$ Diphoton Resonance, arXiv:1512.05332 [INSPIRE].

[10] B. Dutta, Y. Gao, T. Ghosh, I. Gogoladze and T. Li, Interpretation of the diphoton excess at CMS and ATLAS, Phys. Rev. D 93 (2016) 055032 [arXiv:1512.05439] [INSPIRE].

[11] A. Falkowski, O. Slone and T. Volansky, Phenomenology of a $750 \mathrm{GeV}$ Singlet, JHEP 02 (2016) 152 [arXiv:1512.05777] [INSPIRE].

[12] A. Alves, A.G. Dias and K. Sinha, The $750 \mathrm{GeV}$ S-cion: Where else should we look for it?, Phys. Lett. B 757 (2016) 39 [arXiv:1512.06091] [InSPIRE].

[13] J.S. Kim, K. Rolbiecki and R. Ruiz de Austri, Model-independent combination of diphoton constraints at $750 \mathrm{GeV}$, Eur. Phys. J. C 76 (2016) 251 [arXiv: 1512.06797] [INSPIRE].

[14] L. Berthier, J.M. Cline, W. Shepherd and M. Trott, Effective interpretations of a diphoton excess, JHEP 04 (2016) 084 [arXiv: 1512.06799] [INSPIRE].

[15] N. Craig, P. Draper, C. Kilic and S. Thomas, Shedding Light on Diphoton Resonances, arXiv: 1512.07733 [INSPIRE].

[16] K. Harigaya and Y. Nomura, Composite Models for the 750 GeV Diphoton Excess, Phys. Lett. B 754 (2016) 151 [arXiv: 1512.04850] [INSPIRE].

[17] Y. Nakai, R. Sato and K. Tobioka, Footprints of New Strong Dynamics via Anomaly and the 750 GeV Diphoton, Phys. Rev. Lett. 116 (2016) 151802 [arXiv:1512.04924] [INSPIRE].

[18] S. Di Chiara, L. Marzola and M. Raidal, First interpretation of the $750 \mathrm{GeV}$ di-photon resonance at the $L H C$, arXiv: 1512.04939 [INSPIRE]. 
[19] B. Bellazzini, R. Franceschini, F. Sala and J. Serra, Goldstones in Diphotons, JHEP 04 (2016) 072 [arXiv : 1512.05330] [INSPIRE].

[20] S. Matsuzaki and K. Yamawaki, $750 \mathrm{GeV}$ Diphoton Signal from One-Family Walking Technipion, arXiv:1512.05564 [INSPIRE].

[21] L. Bian, N. Chen, D. Liu and J. Shu, A hidden confining world on the $750 \mathrm{GeV}$ diphoton excess, arXiv: 1512.05759 [INSPIRE].

[22] W. Liao and H.-q. Zheng, Scalar resonance at $750 \mathrm{GeV}$ as composite of heavy vector-like fermions, arXiv: 1512.06741 [INSPIRE].

[23] J.M. Cline and Z. Liu, LHC diphotons from electroweakly pair-produced composite pseudoscalars, arXiv:1512.06827 [INSPIRE].

[24] A. Belyaev, G. Cacciapaglia, H. Cai, T. Flacke, A. Parolini and H. Serôdio, Singlets in Composite Higgs Models in light of the LHC di-photon searches, arXiv:1512.07242 [INSPIRE].

[25] C.-W. Chiang, H. Fukuda, K. Harigaya, M. Ibe and T.T. Yanagida, Diboson Resonance as a Portal to Hidden Strong Dynamics, JHEP 11 (2015) 015 [arXiv: 1507.02483] [INSPIRE].

[26] ATLAS collaboration, Search for high-mass diboson resonances with boson-tagged jets in proton-proton collisions at $\sqrt{s}=8 \mathrm{TeV}$ with the ATLAS detector, JHEP 12 (2015) 055 [arXiv: 1506.00962] [INSPIRE].

[27] R. Barbieri and R. Torre, Signals of single particle production at the earliest LHC, Phys. Lett. B 695 (2011) 259 [arXiv: 1008.5302] [INSPIRE].

[28] A.D. Martin, W.J. Stirling, R.S. Thorne and G. Watt, Parton distributions for the LHC, Eur. Phys. J. C 63 (2009) 189 [arXiv:0901.0002] [inSPIRE].

[29] J. Baglio and A. Djouadi, Higgs production at the LHC, JHEP 03 (2011) 055 [arXiv: 1012.0530] [INSPIRE].

[30] E.H. Fradkin and S.H. Shenker, Phase Diagrams of Lattice Gauge Theories with Higgs Fields, Phys. Rev. D 19 (1979) 3682 [InSPIRE].

[31] G. 't Hooft, Naturalness, Chiral Symmetry, and Spontaneous Chiral Symmetry Breaking, lectures given at the Cargése Summer Institute, Cargése France (1979).

[32] S. Dimopoulos, S. Raby and L. Susskind, Light Composite Fermions, Nucl. Phys. B 173 (1980) 208 [INSPIRE].

[33] V.A. Novikov, L.B. Okun, M.A. Shifman, A.I. Vainshtein, M.B. Voloshin and V.I. Zakharov, Charmonium and Gluons: Basic Experimental Facts and Theoretical Introduction, Phys. Rept. 41 (1978) 1 [INSPIRE].

[34] A.G. Cohen, D.B. Kaplan and A.E. Nelson, Counting 4 pis in strongly coupled supersymmetry, Phys. Lett. B 412 (1997) 301 [hep-ph/9706275] [INSPIRE].

[35] M.A. Luty, Naive dimensional analysis and supersymmetry, Phys. Rev. D 57 (1998) 1531 [hep-ph/9706235] [INSPIRE].

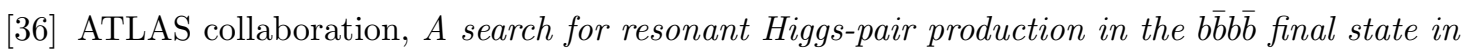
pp collisions at $\sqrt{s}=8 \mathrm{TeV}$, ATLAS-CONF-2014-005 (2014).

[37] CMS collaboration, Search for new resonances in the diphoton final state in the range between 150 and $850 \mathrm{GeV}$ in pp collisions at $\sqrt{s}=8 \mathrm{TeV}$, CMS-PAS-HIG-14-006 (2014). 
[38] ATLAS collaboration, Search for high-mass diphoton resonances in pp collisions at $\sqrt{s}=8 \mathrm{TeV}$ with the ATLAS detector, Phys. Rev. D 92 (2015) 032004 [arXiv:1504.05511] [INSPIRE].

[39] CMS collaboration, Search for a Higgs Boson in the Mass Range from 145 to $1000 \mathrm{GeV}$ Decaying to a Pair of $W$ or $Z$ Bosons, JHEP 10 (2015) 144 [arXiv:1504.00936] [INSPIRE].

[40] ATLAS collaboration, Search for a high-mass Higgs boson decaying to a $W$ boson pair in pp collisions at $\sqrt{s}=8 \mathrm{TeV}$ with the ATLAS detector, JHEP 01 (2016) 032

[arXiv: 1509.00389] [INSPIRE].

[41] ATLAS collaboration, Search for an additional, heavy Higgs boson in the $H \rightarrow Z Z$ decay channel at $\sqrt{s}=8 \mathrm{TeV}$ in pp collision data with the ATLAS detector, Eur. Phys. J. C 76 (2016) 45 [arXiv: 1507.05930] [INSPIRE].

[42] ATLAS collaboration, Search for new resonances in $W \gamma$ and $Z \gamma$ final states in pp collisions at $\sqrt{s}=8 \mathrm{TeV}$ with the ATLAS detector, Phys. Lett. B 738 (2014) 428 [arXiv:1407.8150] [INSPIRE].

[43] ATLAS collaboration, Search for new phenomena in the dijet mass distribution using $p-p$ collision data at $\sqrt{s}=8 \mathrm{TeV}$ with the ATLAS detector, Phys. Rev. D 91 (2015) 052007 [arXiv: 1407.1376] [INSPIRE].

[44] CMS Collaboration, Search for Resonances Decaying to Dijet Final States at $\sqrt{s}=8 \mathrm{TeV}$ with Scouting Data, CMS-PAS-EXO-14-005 (2015).

[45] CMS collaboration, Search for resonances and quantum black holes using dijet mass spectra in proton-proton collisions at $\sqrt{s}=8 \mathrm{TeV}$, Phys. Rev. D 91 (2015) 052009 [arXiv: 1501.04198] [INSPIRE].

[46] D. Goncalves-Netto, D. Lopez-Val, K. Mawatari, T. Plehn and I. Wigmore, Sgluon Pair Production to Next-to-Leading Order, Phys. Rev. D 85 (2012) 114024 [arXiv:1203.6358] [INSPIRE].

[47] G.M. Pruna and T. Robens, Higgs singlet extension parameter space in the light of the LHC discovery, Phys. Rev. D 88 (2013) 115012 [arXiv:1303.1150] [INSPIRE].

[48] T. Robens and T. Stefaniak, Status of the Higgs Singlet Extension of the Standard Model after LHC Run 1, Eur. Phys. J. C 75 (2015) 104 [arXiv:1501.02234] [InSPIRE].

[49] CMS collaboration, Searches for long-lived charged particles in pp collisions at $\sqrt{s}=7$ and $8 \mathrm{TeV}, \mathrm{JHEP} 07$ (2013) 122 [arXiv:1305.0491] [INSPIRE].

[50] M. Krämer et al., Supersymmetry production cross sections in pp collisions at $\sqrt{s}=7 \mathrm{TeV}$, arXiv: 1206.2892 [INSPIRE].

[51] C. Borschensky et al., Squark and gluino production cross sections in pp collisions at $\sqrt{s}=13,14,33$ and 100 TeV, Eur. Phys. J. C 74 (2014) 3174 [arXiv:1407.5066] [InSPIRE].

[52] M. Kawasaki, K. Kohri and T. Moroi, Big-Bang nucleosynthesis and hadronic decay of long-lived massive particles, Phys. Rev. D 71 (2005) 083502 [astro-ph/0408426] [INSPIRE].

[53] M. Kawasaki, K. Kohri, T. Moroi and A. Yotsuyanagi, Big-Bang Nucleosynthesis and Gravitino, Phys. Rev. D 78 (2008) 065011 [arXiv:0804.3745] [INSPIRE].

[54] K. Jedamzik, Big bang nucleosynthesis constraints on hadronically and electromagnetically decaying relic neutral particles, Phys. Rev. D 74 (2006) 103509 [hep-ph/0604251] [INSPIRE]. 
[55] G. Isidori, Y. Nir and G. Perez, Flavor Physics Constraints for Physics Beyond the Standard Model, Ann. Rev. Nucl. Part. Sci. 60 (2010) 355 [arXiv:1002.0900].

[56] K. Hamaguchi, S. Shirai and T.T. Yanagida, Composite messenger baryon as a cold dark matter, Phys. Lett. B 654 (2007) 110 [arXiv:0707.2463] [INSPIRE].

[57] R.M. Godbole, D.J. Miller and M.M. Muhlleitner, Aspects of CP-violation in the $H Z Z$ coupling at the LHC, JHEP 12 (2007) 031 [arXiv:0708.0458] [INSPIRE].

[58] K. Griest and M. Kamionkowski, Unitarity Limits on the Mass and Radius of Dark Matter Particles, Phys. Rev. Lett. 64 (1990) 615 [InSPIRE].

[59] S. Kanemura, S. Matsumoto, T. Nabeshima and N. Okada, Can WIMP Dark Matter overcome the Nightmare Scenario?, Phys. Rev. D 82 (2010) 055026 [arXiv:1005.5651] [INSPIRE].

[60] R.D. Young and A.W. Thomas, Octet baryon masses and sigma terms from an SU(3) chiral extrapolation, Phys. Rev. D 81 (2010) 014503 [arXiv:0901.3310] [INSPIRE].

[61] LUX collaboration, D.S. Akerib et al., Improved Limits on Scattering of Weakly Interacting Massive Particles from Reanalysis of 2013 LUX Data, Phys. Rev. Lett. 116 (2016) 161301 [arXiv: 1512.03506] [INSPIRE].

[62] LZ collaboration, D.S. Akerib et al., LUX-ZEPLIN (LZ) Conceptual Design Report, arXiv: 1509.02910 [INSPIRE].

[63] ATLAS collaboration, Search for resonances in diphoton events with the ATLAS detector at $\sqrt{s}=13 \mathrm{TeV}$, ATLAS-CONF-2016-018 (2016).

[64] CMS collaboration, Search for new physics in high mass diphoton events in $3.3 \mathrm{fb}^{-1}$ of proton-proton collisions at $\sqrt{s}=13 \mathrm{TeV}$ and combined interpretation of searches at $8 \mathrm{TeV}$ and $13 \mathrm{TeV}$, CMS-PAS-EXO-16-018 (2016).

[65] R. Franceschini et al., Digamma, what next?, arXiv:1604.06446 [INSPIRE]. 